\title{
BOUNDED MEAN OSCILLATION WITH ORLICZ NORMS AND DUALITY OF HARDY SPACES
}

\author{
BY JAN-OLOV STRÖMBERG
}

Communicated by A. P. Calderón, July 21, 1976

This paper is a summary of the contents in [5] (with the same title).

$B M O$ was introduced by John and Nirenberg as the space of locally integrable functions $f$ on $\mathbf{R}^{n}$ such that

$$
\int_{Q}\left|f(x)-f_{Q}\right| d x \leqslant A m(Q)
$$

for some constant $f_{Q}$ and for all cubes $Q$ in $\mathbf{R}^{n}$, where $A \geqslant 0$ only depends on $f$. ( $m$ is the Lebesgue measure in $\mathbf{R}^{n}$.) John and Nirenberg [2] have shown that (1) will imply

$$
m\left\{x \in Q ;\left|f(x)-f_{Q}\right|>t\right\} \leqslant C_{1} m(Q) \exp \left(-C_{2} t / A\right), \quad t>0,
$$

with $A$ the same as in (1).

If we assume that $f$ a priori only is a measurable function on $\mathbf{R}^{n}$ and replace (1) by

$$
m\left\{x \in Q ;\left|f(x)-f_{Q}\right|>A^{\prime}\right\}<1 / 2 m(Q),
$$

then it is still true that $f \in B M O$ and (1) holds with $A \leqslant C A^{\prime}$. In fact, a proof similar to that of [2] shows that (1)' implies (2). If the constant $1 / 2$ in (1)' is replaced by a larger number, the result is no longer true.

Let us now assume that the numbers $A$ and $A^{\prime}$ in (1) resp. (1)' are not uniform but depend on $x$ (but not on $Q$ containing $x$ ). We define $M^{\#} f(x)$ resp. $M_{0}^{\#} f(x)$ as the infimum of such $A(x)$ resp. $A^{\prime}(x)$. (2) will now be replaced by

$$
\begin{aligned}
& m\left\{x \in Q ;\left|f(x)-f_{Q}\right|>t\right\} \\
& \quad \leqslant C_{1} m(Q) \exp \left(-C_{2} t / s\right)+m\left\{x \in Q ; M_{0}^{\#} f(x)>s\right\}, \quad s, t>0 .
\end{aligned}
$$

From (3) it follows that $\left\|M_{0}^{\#} f\right\|_{L^{p}}$ is equivalent to $\left\|M^{\#} f\right\|_{L^{p}}, 1<p<\infty$. It was shown by Fefferman and Stein in [1] (under some nonessential restrictions) that

$$
\|M f\|_{L^{p}} \leqslant C\left\|M^{\#} f\right\|_{L^{p}} \quad \text { modulo constants, } 1<p<\infty
$$

AMS (MOS) subject classifications (1970). Primary 30A78, 46E.30.

Key words and phrases. Bounded mean oscillation, Hardy $H$-spaces, maximal functions Orlicz spaces, Riesz transforms. 
where $M$ is the usual Hardy-Littlewood maximal operator. However, (4) is not true for $p=\infty$ since $B M O$ is not contained in $L^{\infty}$. Therefore we are interested in spaces with $M^{\#} f$ in some Orlicz space "near" $L^{\infty}$.

Let $\varphi$ be a nonnegative, increasing, convex function on $\mathbf{R}^{+}(\varphi(0)=0)$, and let $\varphi^{*}$ be its convex conjugate. We define the Orlicz space $L_{\varphi}$ as the space of functions $f$ with norm (see [3])

$$
\|f\|_{L_{\varphi}}=\inf _{\lambda>0} \frac{1}{\lambda}\left[\int_{\mathbf{R}^{n}} \varphi(\lambda|f(x)|) d x+1\right]<\infty .
$$

If $\varphi$ satisfies

$$
\varphi(2 t)<C \varphi(t) \text { for all } t>0 \text {, }
$$

$L_{\varphi}$ is separable with $L_{\varphi^{*}}$ as its dual space.

Now we define $L_{\varphi}^{\#}$ as the space of functions $f$, modulo constants, such that $M^{\#} f \in L_{\varphi}$ with norm $\|f\|_{L_{\varphi}^{\#}}=\left\|M^{\#} f\right\|_{L_{\varphi}}$.

The proof of (4) in [1] works in following more general setting. If $\varphi$ and $\varphi^{*}$ satisfy (5) then (4) holds with the $L^{p}$-norms replaced by $L_{\varphi}$-norms, and then $L_{\varphi}^{\#} \equiv L_{\varphi}$.

We shall now define the Hardy space with Orlicz norm $H_{\varphi}$ as the space of functions $f \in L_{\varphi}$ with the Riesz transforms $R_{i} f, i=1, \ldots, n$, also in $L_{\varphi}$, and with the norm defined by

$$
\|f\|_{H_{\varphi}}=\|f\|_{L_{\varphi}}+\sum_{i=1}^{n}\left\|R_{i} f\right\|_{L_{\varphi}}
$$

As for the $H^{p}$ spaces in $\mathbf{R}^{n}$ (see [4]) there are several equivalent definitions.

If $\varphi$ and $\varphi^{*}$ satisfy (5) then $H_{\varphi} \equiv L_{\varphi}$.

We now come to the main result, which is a generalization of the result of Fefferman and Stein [1] that $B M O$ is the dual space of $H^{1}$.

THEOREM. If $\varphi$ satisfies (5) then $L_{\varphi^{*}}^{\#}$ is the dual space of $H_{\varphi}$. More precisely, there is a dense subset $H_{\varphi}^{0}$ of $H_{\varphi}$ such that (i) for every bounded linear functional $l$ on $H_{\varphi}$ there is a function $g \in L_{\varphi^{*}}^{\#}$ with $\|g\|_{L_{\varphi^{*}}^{\#}}<C\|l\|$ such that

$$
l(f)=\int_{\mathbf{R}^{n}} f(x) g(x) d x
$$

for every $f \in H_{\varphi}^{0}$,

(ii) if $g \in L_{\varphi^{*}}^{\#}$, then

$$
l_{g}(f)=\int_{\mathbf{R}^{n}} f(x) g(x) d x \quad \text { for } f \in H_{\varphi}^{0}
$$

extends to a bounded linear functional on $H_{\varphi}$ and $\left\|l_{g}\right\| \leqslant C\|g\|_{L_{\varphi}^{\#}}$.

The representation is unique, i.e. $I_{g}=0$ if and only if $g$ is constant. 
For the proof we refer to [5]. The only interesting case of the Theorem is when $\varphi$ does not satisfy (5), since otherwise it only says that $L_{\varphi^{*}}^{\#} \equiv L_{\varphi^{*}}$ is the dual space of $H_{\varphi} \equiv L_{\varphi}$.

REMARK. The method of the proof of the Theorem in [5] would work for more general spaces than Orlicz space with the norms defined in terms of distribution functions, for example Lorentz spaces.

Finally, I would like to express my deep gratitude to Professor Lennart Carleson for his advices and interest.

\section{REFERENCES}

1. C. L. Fefferman and E. M. Stein, $H^{p}$-spaces of several variables, Acta Math. 127 (1972), 137-193.

2. F. John and L. Nirenberg, On functions of bounded mean oscillation, Comm. Pure Appl. Math. 14 (1961), 415-426. MR 24 \#A1348.

3. M. A. Krasnosel'skiī and Ya. B. Rutickii, Convex functions and Orlicz spaces, GITTL, Moscow, 1958; English transl., Noorhoff, Groningen, 1961. MR 21 \#5144; 23 \#A4016.

4. E. M. Stein, Singular integrals and differentiability properties of functions, Princeton Univ. Press, Princeton, N. J., 1970. MR 44 \#280.

5. J. O. Strömberg, Bounded mean oscillation with Orlicz norms and duality of Hardy spaces, Institut Mittag-Leffler Report No. 4, 1975, 48 pp. (preprint).

INSTITUT MITTAG-LEFFLER, AURAVÄGEN 17, S-182 62 DJURSHOLM, SWEDEN 